\title{
Prospects for Detection of Gravitational Waves from Intermediate-Mass-Ratio Inspirals
}

\author{
Duncan A. Brown, ${ }^{1,2,3}$ Jeandrew Brink, ${ }^{2}$ Hua Fang, ${ }^{2}$ Jonathan R. Gair, ${ }^{4}$ Chao Li, ${ }^{2}$ Geoffrey Lovelace, ${ }^{2}$ \\ Ilya Mandel, ${ }^{2}$ and Kip S. Thorne ${ }^{2}$ \\ ${ }^{1}$ LIGO Laboratory, California Institute of Technology, Pasadena, California 91125, USA \\ ${ }^{2}$ Theoretical Astrophysics, California Institute of Technology, Pasadena, California 91125, USA \\ ${ }^{3}$ Department of Physics, Syracuse University, Syracuse, New York 13244, USA \\ ${ }^{4}$ Institute of Astronomy, Madingley Road, Cambridge, CB3 OHA, United Kingdom \\ (Received 11 December 2006; revised manuscript received 4 May 2007; published 16 November 2007)
}

\begin{abstract}
We explore prospects for detecting gravitational waves from stellar-mass compact objects spiraling into intermediate mass black holes (BHs) $\left(M \sim 50 M_{\odot}\right.$ to $\left.350 M_{\odot}\right)$ with ground-based observatories. We estimate a rate for such intermediate-mass-ratio inspirals of $\leqslant 1-30 \mathrm{yr}^{-1}$ in Advanced LIGO. We show that if the central body is not a $\mathrm{BH}$ but its metric is stationary, axisymmetric, reflection symmetric and asymptotically flat, then the waves will likely be triperiodic, as for a $\mathrm{BH}$. We suggest that the evolutions of the waves' three fundamental frequencies and of the complex amplitudes of their spectral components encode (in principle) details of the central body's metric, the energy and angular momentum exchange between the central body and the orbit, and the time-evolving orbital elements. We estimate that advanced ground-based detectors can constrain central body deviations from a $\mathrm{BH}$ with interesting accuracy.
\end{abstract}

PACS numbers: 04.80.Nn, 04.25.Nx, 04.30.Db

First generation interferometric gravitational-wave (GW) detectors, such as Laser Interferometer Gravitational-Wave Observatory (LIGO) [1] and Virgo [2], are searching for GWs at or near their design sensitivities. In the next decade, Advanced LIGO (AdvLIGO) [3] and its international partners will increase the volume of the Universe searched a thousandfold or more. The most promising GW sources for this network are the inspiral and coalescence of black hole (BH) and/or neutron star (NS) binaries. Current inspiral searches target sources with total mass $M \lessgtr$ $40 M_{\odot}$ : NS binaries with masses $1-3 M_{\odot}$, BH binaries with masses $3-40 M_{\odot}$, and NS-BH binaries with components in these mass ranges [4,5].

Ultraluminous x-ray observations and simulations of globular cluster dynamics suggest the existence of intermediate-mass black holes (IMBHs) with masses $M \sim$ $10^{2}-10^{4} M_{\odot}$ [6]. The GWs from the inspiral of a NS or stellar-mass BH into an IMBH with mass $M \sim 50-350 M_{\odot}$ will lie in the frequency band of AdvLIGO. These intermediate-mass-ratio inspirals (IMRIs) are analogous to the extreme-mass-ratio inspirals (EMRIs) of $\sim 10 M_{\odot}$ objects spiraling into $\sim 10^{6} M_{\odot} \mathrm{BHs}$, targeted by the planned LISA observatory [7]. We consider IMRIs containing NSs and BHs, as less compact objects (e.g., white dwarfs) are tidally disrupted at frequencies too low to be detectable in AdvLIGO.

If we consider the possibility that the central body of an IMRI (or EMRI) is not a BH, but some other general relativistic object (e.g., a boson star or a naked singularity [8]), then we can quantify the accuracy with which it has the properties predicted for a $\mathrm{BH}$ that: (i) it obeys the $\mathrm{BH}$ no-hair theorem (its spacetime geometry is the Kerr metric, fully determined by its mass and spin), and (ii) its tidal coupling (tide-induced transfer of energy and angular momentum between orbit and body) agrees with $\mathrm{BH}$ predic- tions. Searching for non-BH objects may yield an unexpected discovery.

We report on our initial explorations of the prospects for detecting GWs from IMRIs and probing the properties of IMRIs' central bodies. We report (i) IMRI event rate estimates in AdvLIGO, (ii) estimates of the efficacy of GW template families for IMRI searches, (iii) explorations of the character of the IMRI (EMRI) waves if the central body is not a BH, (iv) generalizations of Ryan's theorem concerning the information about the central body carried by IMRI and EMRI waves, and (v) estimates of the accuracies with which information can be extracted by AdvLIGO from IMRIs.

Event Rates for IMRIs with an IMBH central body. -We (Mandel et al. [9]) estimate that for IMBH spins $\chi \equiv$ angular momentum $/ M^{2} \lesssim 0.3$, the distance (range) $R$ in Mpc to which a network of three $4 \mathrm{~km}$ AdvLIGO detectors could see IMRIs at a network signal-to-noise ratio (SNR) of 8 is

$$
\begin{aligned}
R \approx & {\left[1+\left(\chi^{2} / 2\right)\left(M / 100 M_{\odot}\right)^{1.5}\right] \sqrt{m / M_{\odot}} } \\
& \times\left[800-540\left(M / 100 M_{\odot}\right)+107\left(M / 100 M_{\odot}\right)^{2}\right] .
\end{aligned}
$$

(For IMBHs grown by minor mergers, typical spins will be $\chi \sim \sqrt{m / M} \sim 0.2$, with few if any above $\sim 0.4$.)

Core-collapsed globular clusters are the most likely locations for IMRIs; they may contain an IMBH and a high density of stellar-mass BHs and NSs [6]. Simulations show that it is possible to grow IMBHs with masses up to $M_{\max } \sim 350 M_{\odot}$ through a series of mergers in the core of a cluster [10]. Phinney [11] estimates an upper limit on the IMRI rate in globular clusters as follows: assume each cluster has an IMBH that grows from $\sim 50 M_{\odot}$ to $\sim 350 M_{\odot}$ by capturing objects of mass $m$ in $10^{10} \mathrm{yr}$. Corecollapsed clusters have a space density of $0.7 \mathrm{M} \mathrm{pc}^{-3}$, 
which gives an estimated IMRI rate of $\sim 0.7 \times$ $\left(300 M_{\odot} / \mathrm{m}\right) \times 10^{-10} \mathrm{M} \mathrm{pc}^{-3} \mathrm{yr}^{-1}$. This leads to a limit of $\sim 10$ IMRI detections per year in AdvLIGO.

A kick velocity $V_{\text {kick }}>50 \mathrm{~km} / \mathrm{s}$ will eject the merged $\mathrm{BH}$ from the cluster, placing an upper limit on $m$ of $m / M \lesssim 0.08\left[V_{\text {kick }}\right.$ depends on the symmetric mass ratio $\eta=m M /(m+M)^{2}$ as $V_{\text {kick }} \approx 12000 \eta^{2} \sqrt{1-4 \eta}(1-$ $0.93 \eta) \mathrm{km} / \mathrm{s}$ [12]]. BHs with masses $m \gtrsim 10 M_{\odot}$ will likely merge with the IMBH or be ejected from the core in under $10^{10} \mathrm{yr}$. An estimate based on the dynamics of binary hardening via 3-body interactions yields a rate of one detection per three years for NS-IMBH inspirals or ten detections per year for BH-IMBH inspirals [9]. Optimizing AdvLIGO sensitivity at low frequencies could improve these rates by a factor of $\sim 3$. For Initial LIGO [1], rates are much lower due to lower detector sensitivity and seismic noise below $40 \mathrm{~Hz}$, reducing $M_{\max }$ to $\lesssim 100 M_{\odot}$. We estimate a rate in current detectors of $<1 / 1000 \mathrm{yr}^{-1}$.

Search templates for IMRI waves with an IMBH central body.-Matched filter searches require templates of sufficient accuracy that the mismatch between template and signal does not cause a large loss in event rate. The most accurate IMRI templates currently available come from BH perturbation theory via numerical solution of the Teukolsky equation [13]. Post-Newtonian (PN) templates $[14,15]$ and PN approximations to Teukolsky waveforms [16] are inadequate becuase IMRIs enter the detector frequency band when the binary separation is $r \lessgtr 15 M$ and the PN expansion is poor.

Inspiral waveforms from $\mathrm{BH}$ perturbation theory are known only to first order in $\eta$ plus $\mathrm{O}\left(\eta^{2}\right)$ in radiation reaction. It is important to determine the effect of conservative finite-mass-ratio corrections $\mathrm{O}\left(\eta^{2}\right)$, but tools to study these are not yet in hand. We (Brown [17]) estimate these effects by computing the mismatch (for AdvLIGO) between restricted PN stationary-phase templates containing all known $\eta$ terms, and the same templates linearized in $\eta$ plus $\mathrm{O}\left(\eta^{2}\right)$ radiation reaction (cut off at the IMRI's innermost stable circular orbit); this is the fractional SNR loss due to using templates linearized in $\eta$. Mismatches are computed at each PN order between 1.0 and 3.5 inclusive. For a $1.4 M_{\odot}$ NS $-100 M_{\odot}$ IMBH IMRI, the mismatch is $\$ 30 \%$ for $\chi<0.8$, and $\leqslant 15 \%$ for $\chi<0.3$. For IMRIs with a larger IMBH mass, the mismatch decreases. By allowing the linearized PN waveforms to have mass parameters different from those of the nonlinear PN waveforms, and minimizing the mismatch over these parameters, mismatch falls to less than $10 \%$ in all except the most rapidly spinning cases [17]. Therefore, it is reasonable to expect that Teukolsky waveforms will lose no more than $10 \%$ of the SNR due to linearization in $\eta$ (hence no more than a $30 \%$ loss of event rate). For detection, it will be worthwhile, but not essential, to improve Teukolsky waveforms with nonlinear corrections, but accurate parameter measurement will require improvements.

IMRI and EMRI orbits and waves; triperiodic vs ergodic.-Here we entertain the possibility that the central body is not a $\mathrm{BH}$. We assume its external spacetime geometry is stationary, axially and reflection symmetric, and asymptotically flat (SARSAF) with metric in the form $d s^{2}=-\alpha^{2} d t^{2}+\varpi^{2}(d \phi-\omega d t)^{2}+g_{\theta \theta} d \theta^{2}+g_{r r} d r^{2}$ and all coefficients independent of the Killing time $t$ and axial angle $\phi$. If the spacetime initially is not axisymmetric, rotation will make it nonstationary; then presumably $\mathrm{GW}$ emission drives it to stationarity and axisymmetry on astrophysically small time scales. Almost all stationary, axially symmetric, self-gravitating objects studied observationally or theoretically are reflection symmetric.

A SARSAF solution to the vacuum Einstein equations is determined uniquely by two families of scalar multipole moments: mass moments $M_{0} \equiv M, M_{2}$ (mass quadrupole moment), $M_{4}, \ldots$; and current moments $S_{1}$ (spin angular momentum), $S_{3}, S_{5}, \ldots$ [18]. For the Kerr metric (describing astrophysical $\mathrm{BHs}$ ), the moments are fully determined by the mass $M$ and dimensionless angular momentum $\chi \equiv$ $S_{1} / M^{2}$ via $M_{\ell}+i S_{\ell}=M^{l+1}(i \chi)^{\ell}$; this is the no-hair theorem. LISA plans to measure as many moments as possible, via EMRI waves, and determine the accuracy with which each moment satisfies this Kerr formula; AdvLIGO can do the same for IMRIs.

For EMRIs and IMRIs, the orbiting object moves along an orbit that is nearly a geodesic of the background metric; radiation reaction drives it slowly from one geodesic to another. If the central body is a Kerr BH, then (i) each geodesic has three isolating integrals of the motion: energy $E$, axial angular momentum $L_{z}$, and Carter constant $Q$ (and a fourth, "trivial" integral, the length of the orbit's tangent vector), and (ii) the emitted gravitational waves are triperiodic with $h^{\mu \nu}=\operatorname{Re} \sum_{P k m n} h_{P k m n}^{\mu \nu} e^{i\left(k \Omega_{\theta}+m \Omega_{\phi}+n \Omega_{r}\right) t}$ (for integer values of $k, m, n)$ [19]. Here $P=+, \times$ is the polarization, and the three fundamental frequencies $\Omega_{\theta}$, $\Omega_{\phi}, \Omega_{r}$, in a precise but subtle sense, are associated with the orbital motion in the polar $(\theta)$, azimuthal $(\phi)$, and radial $(r)$ directions. The fundamental frequencies and complex amplitudes evolve with time as the orbit evolves through a sequence of geodesics.

If the Carter constant is lost in SARSAF spacetimes, motion may be ergodic rather than triperiodic, which would make detection of the gravitational waves difficult. Guéron and Letelier [20] have used Poincaré maps to search for ergodic geodesics in the static $\left(S_{\ell}=0\right)$ ErezRosen metric and we (Gair et al. [21]) have carried out similar studies for a variant of the stationary $\left(S_{\ell} \neq 0\right)$ Manko-Novikov metric [22]. Both of these metrics have arbitrary mass quadrupole moment $M_{2}$, and higher order moments fixed by $M_{2}, S_{1}$, and $M$. The Poincaré maps in these spacetimes reveal that there are geodesics at very small radii $r \sim$ few $M$ that appear ergodic, but none at large radii. We [21] found such geodesics only for oblate $\left(M_{2}<0\right)$ perturbations of spacetimes with spin, but in the Erez-Rosen case [21], ergodicity appears only for prolate $\left(M_{2}>0\right)$ perturbations. Radiation reaction drives the evolution of energy and angular momentum in a way that 
makes it unlikely that the apparently ergodic geodesics could be encountered in the course of an inspiral [21]. For the apparently nonergodic (integrable) geodesics, the spatial coordinates are multiperiodic functions of Killing time $t$ to a numerical accuracy of $10^{-7}$, and a general argument [23] based on the structure of the gravitational propagator shows that their gravitational waves will have the same kind of triperiodic form as for Kerr BHs.

There are three possible explanations for the presence of large-radius orbits that appear integrable and small-radius orbits that appear ergodic in the same spacetime: (i) The orbits are actually integrable and actually ergodic, respectively. (ii) All the orbits are ergodic, but at large radii they appear integrable to numerical accuracy because of the Kolmogorov-Arnol'd-Moser theorem [24]. (iii) All the orbits are integrable, but at small radii they are made to appear chaotic by some ill-understood numerical instability. It is important to learn which is the case, but for EMRI and IMRI wave observations, apparent integrability (or ergodicity) has the same observational implications as actual integrability (or ergodicity).

Information carried by IMRI (EMRI) waves; generalizing Ryan's theorem. - What information about the central body is encoded in the waveforms? We assume the waveforms to be triperiodic. In principle, a large amount of information can be encoded in the time evolution of the waves' three fundamental frequencies $\Omega_{\theta}(t), \Omega_{\phi}(t), \Omega_{r}(t)$, and their complex amplitudes $h_{P k m n}(t)$. It has been speculated that these encode, fully and separably, the values of all the central body's multipole moments $\left\{M_{\ell}, S_{\ell}\right\}$ and hence its metric [25], the rates at which the orbiting object's tidal pull deposits energy and angular momentum into the central body, $\dot{E}_{\text {body }}$ and $\dot{L}_{\text {body }}$ (tidal coupling) [26], and the orbit's semilatus rectum $p(t)$, eccentricity $e(t)$, and inclination angle $\iota(t)$ (which carry the same information as the isolating integrals) [27]. This has been suggested by a special case studied by Ryan [25] (nearly circular, nearly equatorial orbits). A trivial extension of Ryan's theorem [27] leads to the following algorithm for extracting information from the waves. Observe the time-evolving modulation frequencies as functions of the time-evolving fundamental frequency $f=\Omega_{\phi} / \pi$. From these, deduce the functions $\Omega_{A}\left(\Omega_{\phi}\right)$ and then $\Omega_{A}(v)$ for $A=\theta, r$; expand in powers of $v \equiv\left(M \Omega_{\phi}\right)^{1 / 3} \simeq$ (orbital velocity); and read out the moments (redundantly) from the two expansions. Then, knowing the moments and thence the metric, use the geodesic equation to deduce $p(t)$ from $\Omega_{\phi}(t)$ and use wave-generation theory to deduce $e(t)$ and $\iota(t)$ from particular modulation amplitudes, $h_{P k m n}(t)$.

We have generalized Ryan's theorem to strongly elliptical but nearly equatorial orbits ( $\mathrm{Li}$ [23]), to include tidal coupling ( $\mathrm{Li}$ and Lovelace [27]), and are working on further generalizations. For strongly elliptical but nearly equatorial orbits the three fundamental frequencies are independent of $\iota$ at first order. We expand these frequencies $\Omega_{A}\left(M_{\ell}, S_{\ell}, e, p\right)$ (with $\left.A=\theta, \phi, r\right)$ in powers of $1 / p$, with coefficients that depend on $e$ and the moments. Suppose we observe a series of $2 N+1$ values of $\Omega_{\theta}, \Omega_{\phi}, \Omega_{r}$ ) (for any integer $N$ ) during the course of an inspiral. This gives us $6 N+3$ numbers, from which we can read off (via an algorithm based on our expansions of the fundamental frequencies [23]): (i) the time evolution of $e(t)$ and $p(t)$ $(2 N+1$ values of each), (ii) the lowest $N+1$ mass moments, and (iii) the lowest $N$ current moments. By observing the evolving amplitudes of the orbital-precessioninduced modulations encoded in $h_{P k m n}$, we can recover the time evolution of $\iota$. Hence, in principle, we have a full description of the spacetime. In practice the methods of extracting the information are likely to be quite different from these algorithms.

In the absence of tidal coupling Ryan demonstrated that, for a nearly circular, nearly equatorial orbit, the central body's moments are encoded not only in the waves' modulations, but also in the phase evolution of the waves' dominant harmonic $f=\Omega_{\phi} / \pi$. We have extended this analysis to deduce the power being deposited in the central body by tidal coupling, $\dot{E}_{\text {body }}$ [27]. We assume the moments and metric have been deduced from the precessional modulations and then use deviations from the Ryantheorem phase evolution to deduce $\dot{E}_{\text {body }}$. Following Ryan, we quantify the waves' phase evolution by $\Delta N(t) \equiv$ $f^{2} / \dot{f}=d$ (number of wave cycles) $/ d \ln f$. From this definition of $\Delta N$, we infer the rate of change of orbital energy: $\dot{E}_{\text {orb }}=\left(d E_{\text {orb }} / d \Omega_{\phi}\right)\left(\Omega_{\phi}^{2} / \pi \Delta N\right)$. All (time-evolving) quantities on the right side can be deduced from observation plus the geodesic equation (for $d E_{\text {orb }} / d \Omega_{\phi}$ ). From the deduced metric and the frequency $f(t)$ we can compute the power radiated to infinity $\dot{E}_{\infty}$; and then by energy conservation we can deduce the power being deposited in the central body $\dot{E}_{\text {body }}=-\dot{E}_{\text {orb }}-\dot{E}_{\infty}$ [27]. We can also infer the angular momentum transferred tidally to the central body, $\dot{L}_{\text {body }}$, via $\dot{L}_{\text {body }}=\dot{E}_{\text {body }} / \Omega_{\phi}$ (valid for nearly circular, nearly equatorial orbits).

The above argument assumes that we can compute $\dot{E}_{\infty}$ without knowing the boundary conditions of the inspiralinduced metric perturbation at the central body, since we do not know the nature of the central body a priori. For highly compact central bodies (those deep inside the perturbing field's "effective potential") this is true to high but not complete accuracy. The effect of boundary conditions at the central body on the inspiral phase evolution is communicated outward to infinity mainly at low frequencies (the orbital frequency and its low-order harmonics), and these perturbations have great difficulty penetrating through the effective potential. If the spacetime metric is Kerr, we have shown that the influence of the inner boundary condition on the energy radiated to infinity is $\delta \dot{E}_{\infty} \sim v^{10} \dot{E}_{\infty}$ [27]-five orders smaller in the linear velocity $v$ than the tidal coupling $\dot{E}_{\text {body }} \sim v^{5} \dot{E}_{\infty}$ [28]. Thus, to high accuracy we can deduce $\dot{E}_{\infty}$ and then $\dot{E}_{\text {body }}$ from observations, without knowing the body's precise nature. 
Measurement accuracies for AdvLIGO. - We have estimated how accurately AdvLIGO, via IMRI waves, can constrain deviations of the central body's quadrupole moment $M_{2}$ (Brown [17]) and tidal coupling $\dot{E}_{\text {body }}$ (Fang [29]) from those of a Kerr BH. Absent the true waveforms, we used PN waveforms as signals and templates. This introduces systematic error, but we believe our results indicate the accuracies achievable. Our source is the circular inspiral of a neutron star into a $100 M_{\odot}$ IMBH (under the assumption that radiation reaction has circularized the orbit [9]). The orbit is inclined to the hole's equatorial plane, to produce a modulation crucial for breaking degeneracy between the IMBH spin $\chi$ and $M_{2}$ and $\dot{E}_{\text {body }}$.

To investigate $M_{2}$, we used templates accurate to $3.5 \mathrm{PN}$ order in phase evolution [15] and 1.5PN in spin-orbit coupling [14], which include the quadrupole-monopole interaction [30] in both the phase and the precessional modulation, and numerically mapped the ambiguity function of these signals. For a NS-IMBH IMRI $\left(M_{2}=\right.$ $-\chi^{2} M^{3}$ ) with spin $\chi=0.8$ and SNR $\sim 10$, we found AdvLIGO measurement errors $\Delta \ln M \sim 0.006, \Delta \ln \chi \sim$ 0.02 , and $\Delta \ln M_{2} \sim 0.6$. If the IMBH spin is $\chi=0.3$, the error increases to $\Delta \ln M \sim 0.01, \quad \Delta \ln \chi \sim 0.3$, and $\Delta \ln M_{2} \sim 2$. Measurement accuracy depends strongly on binary orientation; larger precessional modulation reduces the errors [17].

We model tidal coupling as $\dot{E}_{\text {body }} \equiv \epsilon \dot{E}_{\mathrm{BH}}$, where $\dot{E}_{\mathrm{BH}}$ is the energy flow into a Kerr $\mathrm{BH}$ [28] and seek to measure deviations parametrized by $\epsilon$. We constructed precessing waveforms [31], with orbital inspiral phase given by the 3.5 PN approximation of the Teukolsky waveforms [16], and modulation linearized in inclination angle [32]. We restricted inclination angles to $\iota<\pi / 4$, fixed the direction to the source and the central body's spin orientation, and used the Fisher matrix to estimate parameter measurement accuracies. For a BH central body with spin $\chi=0.8$ and $\mathrm{SNR}=10$, we could measure $\epsilon$ to $\Delta \ln \epsilon \sim 1$ to 2 , increasing to $\Delta \ln \epsilon \sim 30$ at $\chi=0.3$.

While these errors are larger than hoped, (i) the quadrupole moment $\left|M_{2}\right|$ of a boson star with $\chi=0.3$ is expected to be in the range 15 to $100 \chi^{2} M^{3}$ [8], so AdvLIGO could readily identify such a central body, (ii) for small spins $\dot{E}_{\mathrm{BH}} \simeq-\frac{1}{4} \chi v^{5} \dot{E}_{\infty}$, and hence for $\chi=$ 0.3 , the accuracy of measuring tidal coupling is $\Delta \dot{E}_{\text {body }} \sim$ $30 \times 0.001 \dot{E}_{\infty}$, i.e., $3 \%$ of the power radiated to infinity, an interesting accuracy for central bodies with anomalously large $\dot{E}_{\text {body }}$, and (iii) observing an IMRI in each of the three AdvLIGO detectors increases the accuracy of parameter estimation quoted by a factor of $\sqrt{3}$; including additional detectors, e.g., advanced Virgo, could improve this further. In practice, parameter estimation will be pursued using Markov chain Monte Carlo techniques [17,33].

Our results suggest that AdvLIGO could verify with modest but interesting accuracy that an IMRI's central body is a Kerr BH, and perform searches for non-Kerr objects. AdvLIGO's accuracies for probing the central body are far worse than LISA's (as expected, due to the thousandfold fewer wave cycles), but AdvLIGO is likely to be operational some years before LISA. Its studies of central bodies will be a valuable precursor to LISA's EMRI science, and might possibly yield a big surprise.

We are grateful to Y. Chen, T. Creighton, C. Cutler, S. Drasco, C. Miller, Y. Pan, and S. Phinney for discussions. This work was supported in part by NSF Grants PHY-0099568 and PHY-0601459, NASA Grant NNG04GK98G, a grant from the Brinson Foundation, and NSF cooperative agreement PHY-0107417.

[1] B. C. Barish and R. Weiss, Phys. Today 10, No. 10, 44 (1999).

[2] F. Acernese et al., Classical Quantum Gravity 23, S635 (2006).

[3] P. Fritschel, arXiv:gr-qc/0308090.

[4] B. Abbott et al., Phys. Rev. D 72, 082001 (2005).

[5] B. Abbott et al., Phys. Rev. D 73, 062001 (2006).

[6] M. C. Miller and E. J. M. Colbert, Int. J. Mod. Phys. D 13, 1 (2004).

[7] J. R. Gair et al., Classical Quantum Gravity 21, S1595 (2004).

[8] F. D. Ryan, Phys. Rev. D 55, 6081 (1997).

[9] I. Mandel, D. A. Brown, J.R. Gair, and M. C. Miller, arXiv:astro-ph/0705.0285 (to be published).

[10] R. M. O'Leary et al., Astrophys. J. 637, 937 (2006).

[11] E. S. Phinney (private communication).

[12] J. A. González et al., Phys. Rev. Lett. 98, 091101 (2007).

[13] S. A. Hughes, Phys. Rev. D 64, 064004 (2001).

[14] L. E. Kidder et al., Phys. Rev. D 47, R4183 (1993).

[15] L. Blanchet et al., Phys. Rev. D 65, 061501(R) (2002).

[16] H. Tagoshi et al., Phys. Rev. D 54, 1439 (1996).

[17] D. A. Brown (to be published).

[18] R. Hansen, J. Math. Phys. (N.Y.) 15, 46 (1974).

[19] S. Drasco and S.A. Hughes, Phys. Rev. D 69, 044015 (2004).

[20] E. Guéron and P. S. Letelier, Phys. Rev. E 66, 046611 (2002).

[21] J.R. Gair, C. Li, and I. Mandel, arXiv:gr-qc/0708.0628 [Phys. Rev. D (to be published)].

[22] V.S. Manko and I.D. Novikov, Classical Quantum Gravity 9, 2477 (1992).

[23] C. Li (to be published).

[24] M. Tabor, Chaos and Integrability in Nonlinear Dynamics (John Wiley \& Sons Inc., New York, 1989).

[25] F. D. Ryan, Phys. Rev. D 52, 5707 (1995).

[26] H. Fang and G. Lovelace, Phys. Rev. D 72, 124016 (2005).

[27] C. Li and G. Lovelace, arXiv:gr-qc/0702146.

[28] H. Tagoshi et al., Prog. Theor. Phys. 98, 829 (1997).

[29] H. Fang, Ph.D. thesis, Caltech, Pasadena, CA.

[30] E. Poisson, Phys. Rev. D 57, 5287 (1998).

[31] T. A. Apostolatos et al., Phys. Rev. D 49, 6274 (1994).

[32] M. Shibata et al., Phys. Rev. D 51, 1646 (1995).

[33] C. Rover et al., Classical Quantum Gravity 23, 4895 (2006). 